MUZIKOLOŠKI ZBORNIK • MUSICOLOGICAL ANNUAL XL

UDK 73/76(497.4)

\title{
Damjan Prelovšek
}

France-Stele kunstgeschichtliches Institut der Forschungszentrum der Slowenischen

Akademie für Wissenschaften und Künste, Ljubljana

Umetnostnozgodovinski inštitut Franceta Steleta Znanstvenoraziskovalnega centra

Slovenske akademije znanosti in umetnosti, Ljubljana

\section{Slowenische bildende Kunst und ihre Einbindung in den mitteleuropäischen Raum}

\section{Slovenska likovna umetnost in njena vpletenost $\mathrm{v}$ srednjeevropski prostor}

ZUSAMMENFASSUNG

Der Beitrag behandelt die traditionelle Spannung in der slowenischen bildenden Kunst zwischen dem Mittelmeerraum und dem Norden, wobei mit dem letzteren mehr oder weniger Mitteleuropa gemeint ist. Wenn man doch versucht, diesen ziemlich unpräzisen geographisch-kulturellen Begriff mindestens ein wenig abzugrenzen, kann man sagen, daß es dabei um die Länder geht, die dem westeuropäischen Kulturkreis gehören, jedoch in mancher Hinsicht auch darüber hinaus gehen, ohne ihren westeuropäischen zivilisatorischen Wurzeln untreu zu werden, wobei sie in geographischer Hinsicht nicht zu West-, Süd-, Nord- oder Osteuropa gehören. Im Falle Sloweniens waren das vor allem Süddeutschland, Böhmen und Österreich. In der Geschichte hatten Einflüsse aus diesen Ländern verschiedene Auswirkungen auf den slowenischen ethnischen Raum, was jeweils vor allem von den momentanen dynastischen und kirchlichen Verbindungen abhängig war. Eine stärkere und längerfristige Anlehnung an die mitteleuropäische Kultur hatte jedes Mal auch ein mehr oder weniger ausgeprägtes Zurückkehren zu Italien zur Folge. Nach der Konsolidierung der absolutistisch geregelten zentralisierten Monarchie überwog in Slowenien endgültig der Einfluß Wiens und dadurch derjenige der mitteleuropäischen Kultur, die aber auch selbst noch viele mediterrane Elemente beinhaltete. Jedoch gingen diese Einflüsse nie
POVZETEK

Prispevek obravnava tradicionalno razpetost slovenske likovne umetnosti med Mediteran in sever, pri čemer gre v zadnjem primeru bolj ali manj za Srednjo Evropo. Če skušamo ta sicer dokaj ohlapni geografsko-kulturni pojem vsaj nekoliko zamejiti, potem lahko rečemo, da gre za dežele, ki pripadajo zahodnoevropskemu kulturnemu krogu, vendar ga v marsičem tudi presegajo, čeprav se ne oddaljujejo od svojih zahodnoevropskih civilizacijskih korenin, medtem ko $\mathrm{v}$ zemljepisnem pogledu ne sodijo v zahodno, južno, severno in vzhodno Evropo. V primeru Slovenije so bile to predvsem južna Nemčija, Češka in Avstrija. V zgodovini so vplivi teh dežel različno posegali v slovenski etnični prostor, kar je bilo odvisno zlasti od trenutnih dinastičnih in cerkvenih povezav. Močnejši in dolgotrajnejši naslon na srednjeevropsko kulturo je vsakič izzval tudi bolj ali manj izrazito vračanje $\mathrm{k}$ Italiji. $\mathrm{Z}$ utrditvijo absolutistično urejene centralizirane monarhije je v Sloveniji dokončno prevladal vpliv Dunaja in $z$ njim srednjeevropske kulture, ki pa je tudi sama nosila še veliko mediteranskih elementov. Vendar pa ti vplivi nikoli niso potekali samo enosmerno iz severa proti jugu, ampak se je tudi nekaterim slovenskim umetnikom posrečilo uveljaviti v širšem srednjeevropskem prostoru, kar velja za generacijo slovenskih impresionistov (Jakopič, Grohar, Jama, Strnen) in še zlasti za arhitekta Jožeta 
MUZIKOLOŠKI ZBORNIK - MUSICOLOGICAL ANNUAL XL

nur in einer Richtung, von Norden nach Süden, sondern gelang es auch einigen slowenischen Künstlern, sich im weiteren mitteleuropäischen Raum zu etablieren, was für die Generation der slowenischen Impressionisten (Jakopič, Grohar, Jama, Sternen) und vor allem für den Architekten Jože Plečnik gilt, der entscheidend die österreichische und die tschechische Kunst beeinflußte. Nach dem Zweiten Weltkrieg verblaßten ein bißchen die Kontakte zu Mitteleuropa, nicht zuletzt auch wegen der politischen Gründe. Die slowenischen Künstler suchten sich Vorbilder eher in den westeuropäischen Kulturzentren und in Nordamerika, aber in der letzten Zeit kommt es langsam wieder auch zur Erneuerung der traditionellen Verbindungen mit den einmal nahen ausländischen Städten. Inwieweit die slowenische Kunst überhaupt noch in Mitteleuropa verankert ist und inwieweit das letztere noch sich selbst treu geblieben ist, wird aber die Zeit zeigen.
Plečnika, ki je odločilno posegel $\mathrm{v}$ avstrijsko in češko umetnost. Po drugi svetovni vojni so povezave s Srednjo Evropo nekoliko zamrle, nenazadnje tudi zaradi političnih razlogov. Slovenski umetniki so se raje zgledovali pri zahodnoevropskih kulturnih središčih in pri severni Ameriki, vendar v zadnjem času počasi spet prihaja do obnavljanja tradicionalnih povezav z nekdanjimi bližjimi tujimi mesti. Koliko pa je slovenska umetnost sploh še zasidrana v Srednji Evropi in koliko je slednja še zvesta sama sebi, pa bo pokazal čas.

Die slowenische Kultur und mit ihr auch die bildende Kunst stand immer in der Spannung zwischen Italien und dem europäischem Norden, wobei der letztere in etwa dem entsprach, was wir heute unter dem Begriff Mitteleuropa verstehen. Abgesehen davon, was Mitteleuropa überhaupt ist, beziehungsweise, welche Länder es umfaßt, ist für unsere Behandlung mindestens eine ganz generelle Definition davon notwendig. Zur bildenden Kunst des sogenannten Mitteleuropa gehört grundsätzlich alles, was nicht ausgesprochen westeuropäisch ist, aber doch Merkmale der westeuropäischen christlichen Zivilisation aufweist. Wieviel konnten der Mittelmeerraum oder der Norden zur slowenischen Kunst beitragen, war von verschiedenen Faktoren abhängig, unter welchen auch die nationale Zugehörigkeit der Feudalherren keine vernachlässigbare Rolle spielte. Die meisten Künstler kamen nämlich zu uns durch verschiedene Beziehungen der führenden Adelsfamilien, deren Sitze in den meisten Fällen außerhalb des slowenischen ethnischen Territoriums waren. Durch die Formierung der historischen Länder differenzierte sich auch das Bild der Kunst, so daß es nicht möglich ist, über einen einheitlichen Strom der Einflüsse zu sprechen. Das slowenische Küstenland und das Görzer Gebiet gehörten immer eindeutig zum italienischen Einflußbereich und reagierten kaum jemals auf die mitteleuropäischen Initiativen, wohingegen der Steiermärker und der Kärntner Teil Sloweniens für die letzteren sehr empfänglich waren. Der zentrale Teil Sloweniens, d.h. das gesamte Territorium Krains, schöpfte aber aus den beiden Richtungen. Die kirchliche Abgrenzung zwischen Salzburg und Aquileia verlor langsam an Bedeutung und spielte - mindestens hinsichtlich der Kunst - eine untergeordnete Rolle. Die einzige Ausnahme dabei war die mittelalterliche Kirchenarchitektur in Unterkrain, die mit ihrer betonten mediterranen Tektonik ziemlich lange den Aquileier Mustern folgte.

Im Mittelalter kamen in unsere Länder vom Westen her viele wandernde lombardische Steinmetzen und friaulische Maler, die auf eine volkstümliche Weise die italienischen Neuheiten verbreiteten. Trotzdem orientierte sich die Architektur größtenteils am Geschehen in den deutschen Ländern, vor allem in Bayern, aus dem, wie 
gesagt, die meisten dynastischen Verbindungen stammten. Im 13. Jahrhundert kam allerdings ein großer Teil des slowenischen Territoriums unter die tschechische Krone von Premisl Otokar II., aber seine Herrschaft war von zu kurzer Dauer, um in der Kunst bleibendere Spuren zu hinterlassen. Mit dem Aufstieg der Grafen von Cilli und mit ihrer europäischen Politik kam es dazu, daß die Künstler begannen, sich mit mehr Ambition in Mitteleuropa Vorbilder zu suchen. Als ein bescheidenes, jedoch außerordentlich hochqualitatives Echo der französischen, wahrscheinlich über Deutschland gekommenen Kathedralenkunst entstand um das Jahr 1400 die Marienkapelle der Abteikirche in Cilli (Celje). Der Schwerpunkt des kreativen Schaffens wurde damals aber von Süddeutschland nach Böhmen verschoben, wo die Prager St.Veits-Kathedrale, eine der größten Errungenschaften der mitteleuropäischen Gotik, von der Dombauhütte Peter Parlers gebaut wurde. Bei diesem Bau bildete sich eine Reihe der Baumeister und Steinmetzen aus, die später auch nach Slowenien kamen. Am aktivsten waren sie in Pettau (Ptuj) und seiner Umgebung, worüber insbesondere das alte Presbyterium der Pfarrkirche in Zgornja Hajdina (von ca. 1390) und die dreischiffige Marienkirche in Ptujska gora (von ca. 1400), eine Perle der slowenischen Gotik, zeugen. Gleichzeitig entstanden einige außergewöhnliche Statuen, die zum Gipfel des sogenannten europäischen "Weichen Stils" gehören. Daß es sich dabei um eine breitere Welle der Parlerkunst handelte, zeigen deren Echos im Chor des alten Laibacher (Ljubljanaer) Domes, wie auch im Schiff der Pfarrkirche in Krainburg (Kranj), in den Kartäuserkirchen in Pleterje und Žiče und in der Propsteikirche und dem Dominikanerkloster in Pettau.

In der Spätgotik begann sich auch in Slowenien der aus den deutschen Ländern kommende Typ der Hallenkirche zu etablieren, der sich aber wegen der Nähe Italiens nie völlig durchsetzte. Obwohl das 15. Jahrhundert auf dem Gebiet der Malerei eine goldene Ära der Freskos war, derer Autoren größtenteils aus Friaul und aus Görz kamen, sind nicht gerade viele gotische Tafelmalereien erhalten geblieben. Die Werke von höherer Qualität waren in der Regel aus deutschen Ländern importiert, so zum Beispiel das Marientriptychon aus der Pettauer Propsteikirche, das um 1460 vom Salzburger Bürger und anerkannten Künstler Konrad Laib gemalt wurde, und der ehemalige Hauptaltar der Krainburger Pfarrkirche. Den Krainburger Altar mit den Aquileier Patronen der Pfarrei bestellten die Pilger aus Krainburg zwischen den Jahren 1500 und 1510 in Köln, am wahrscheinlichsten in der Werkstatt vom so genannten Meister des Bartholomäus-Altars. Heute ist es der Stolz der Österreichischen Galerie Belvedere in Wien.

Die osmanischen Einfälle und die religiösen und sozialen Unruhen führten Ende des 15. Jahrhunderts zu einer Stagnation, aus der die slowenischen Länder lange keinen Weg finden konnten. Das 16. Jahrhundert verging im Zeichen der Befestigung der Städte und Schlösser. Die besten Experten waren die Italiener. Nach der Errichtung Innerösterreichs kamen sie über die steirische Stadt Graz auch in die slowenischen Länder. Für ein Aufblühen der Renaissance und des Manierismus gab es in den ökonomisch schwierigen Zeiten keine richtigen Bedingungen. Unter den wichtigeren Werken der ersten Hälfte des 16. Jahrhunderts muß man die Bemalung der Pilgerkirche der hll. Primus und Felician oberhalb von Stein (Kamnik) (1504) erwähnen. Die 
Freskos sind Arbeit eines unbekannten Künstlers aus dem Norden, der auch die damalige italienische Malerei kannte. Dank dem Laibacher Bischof Krištof Rauber entwickelte sich die Hauptstadt Krains für eine kurze Zeit zum Zentrum des Humanismus in Slowenien. Bei der Einrichtung seiner Residenz in Oberburg (Gornji grad) engagierte der Bischof den in Schwaben ausgebildeten Bildhauer Osbalt Kittel, der für die neue Kapelle in der dortigen Kirche im Jahre 1527 unter anderem den St. Andreas-Altar gestaltete. Der Protestantismus brachte allerdings keine bedeutenden Schöpfungen der bildenden Kunst hervor, dafür errang man aber in der Buchkultur mit Dalmatins Übersetzung der Bibel, die mit den Holzschnitten der deutschen Graphikkünstler ausgestattet war, ein beneidenswertes Niveau.

Die ganze Zeit bis zum Ende des 17. Jahrhunderts gab es vereinzelte Versuche, das Land aus der Lethargie zu wecken, in der es wegen der unaufhörlichen osmanischen Gefahr steckte. Um das Jahr 1600 malte zum Beispiel ein wandernder mitteleuropäischer Maler nach italienischen und niederländischen graphischen Vorlagen die Decke für den Hauptsaal "Stara grofija" (Alte Grafschaft) in Cilli. Die vor allem in Bayern entstandenen graphischen Darstellungen der Werke zeitgenössischer europäischer Meister waren eine Quelle der Inspiration für die heimischen Maler, die aus ihnen mit größerem oder kleinerem Erfolg einzelne Motive kopierten; sie dienten aber auch als Vorlagen bei der Bemalung von Schloßsälen und Kirchen. Unter den wenigen größeren Kirchenbauten sollte man mindestens die Marienwallfahrtskirche in Puščava pod Pohorjem erwähnen. Errichtet wurde die Kirche von den Mönchen des Klosters St. Paul in Kärnten, die sie Anfang der siebziger Jahre des 17. Jahrhunderts auch vergrößerten und ihr nach dem Vorbild des Salzburger Domes einen Dreikonchenabschluß anbauten. In den achtziger Jahren des 17. Jahrhunderts gründete der Polyhistor Johann Weikhard Valvasor am Schloß Bogenšperk eine bedeutende graphische Werkstatt, die eine Art Auftakt für das Aufblühen des Laibacher Barocks am Anfang des neuen Jahrhunderts darstellte. Anders wie später in der ausgesprochen italienisch ausgerichteten Academia operosori überwog in Valvasors Kreis der Geschmack der nördlichen bildenden Kunst. In seiner Nähe wirkte auch der noch heute unerforschte niederländische Maler Almanach, der in der letzten Zeit in der slowenischen Kunstgeschichtsschreibung für großes Aufsehen sorgt.

Auf eine ganz andere Weise blühte die Barockkunst in der Steiermark, denn Graz behielt die führende Position auch nach dem Ende Innerösterreichs. Die günstigen Verhältnisse zogen eine Reihe ausländischer Maler in die Stadt, unter ihnen die Bayern Hans Adam Weissenkirchner (1646-1695) und Franz Josef Ignatz Flurer (1688-1742), die beide auch auf dem slowenischem Territorium wirkten. Generell kann man aber sagen, daß in unserer Barockmalerei der führende Platz der Steiermark gehörte. Unter den Künstlern Krains errang größeren Ruhm Franz Karl Remb (1675-1718) aus Radmannsdorf (Radovljica), der es zu einem der hochangesehenen Wiener Maler seiner Zeit schaffte und einige Jahre vor seinem Tod zum Direktor der Sammlungen des Fürsten von Liechtenstein wurde. Auf eine ähnliche Weise machte sich Franc Andrej Šega (1711-1787) aus Rudolfswert (Novo mesto) in der Hauptstadt Bayerns einen Namen als Medailleur. Von den wichtigeren Ausländern, die im Barock im Land Krain malten, kann man nicht das umfangreiche Gesamtwerk von Martin Johann Schmidt 
MUZIKOLOŠKI ZBORNIK • MUSICOLOGICAL ANNUAL XL

(1718-1801) aus dem österreichischen Krems unbeachtet lassen, dessen Werke in Laibach, Velesovo und Oberburg erhalten blieben.

In der steirischen Baukunst herrschten weiche gewellte Formen vor, die über Wien und Graz aus Süddeutschland und Böhmen in unsere Länder kamen. Die Wände der Kirchenräume gingen allmählich in eine durchsichtige Membran über, die sich mit ihrer Deckenbemalung illusionistisch in Richtung Himmel ausbreitete. Die Krainer Architektur, verankert ziemlich fest in der mediterranen Tektonik, erlebte nie eine solche Öffnung gegenüber den mitteleuropäischen Initiativen.

Die entscheidende Wende in Richtung Mitteleuropa erlebte Slowenien nach der Mitte des 18. Jahrhunderts durch die absolutistische Politik der Habsburger. Das neugegründete Theresianum in Wien versetzte den Standort der Adelsausbildung in das Zentrum des Reiches, was auch die Kunstideale der wichtigsten Gesellschaftsschicht der Kunstauftraggeber änderte. Die teure Führung des Krieges gegen Frankreich und Preußen zwang zum allgemeinen Sparen. Das zeigte sich in einem kleineren allgemeinen Interesse für Kunst und in einem rationelleren Verhältnis zur Architektur. Damals endeten die Reisen der Gehilfen quer durch Italien, und die künftigen Baumeister begannen, ihre an der Wiener Akademie angeeigneten Kenntnisse in Deutschland und Böhmen zu vervollkommnen. Nachdem einige österreichische Baumeister gekommen waren, endete die Periode des fruchtbaren Dialogs mit dem Mittelmeerraum. Die slowenische bildende Kunst wurde völlig mitteleuropäisch, obwohl sie in einen viel kleinere Rahmen gezwängt wurde. Der Wiener Hof bemühte sich allerdings noch immer, dem französischen zu folgen, aber das konnte man im Randgebiet des Staates kaum spüren. Die zentralisierten und bürokratisierten Kunstinstitutionen des Staates inthronisierten den bürgerlichen Biedermeierstil als eine Antwort auf den glänzenden französischen "style Empire". Die Reichweite der slowenischen Kunst erstreckte sich danach nur noch bis Wien. Zu Professoren an der Wiener Akademie der bildenden Künste wurden in dieser Zeit der etwas bescheidenen Kunst der Landschaftsmaler Lovro Janša (1749-1812) aus Breznica bei Radmannsdorf und der Görzer Franc Kavčič (1755-1828), der mit seinen großformatigen Kompositionen als einer der vornehmeren Vertreter des mitteleuropäischen Klassizismus gilt. Andererseits etablierte sich in der österreichischen Baukunst des Vormärz der Laibacher Jožef Šemerl (Schemerl) von Leytenbach (1754-1844), vor allem als geschickter Organisator und Experte für Wasserbauten.

Zum Teil änderten sich die Verhältnisse nach der Märzrevolution, als in Österreich eine größere künstlerische Freiheit entstand, auf die die slowenischen Länder nicht vorbereitet waren. Gegen Ende des 19. Jahrhunderts schafften die Brüder Janez (1850 1889) und Jurij Šubic (1855-1890) den Sprung von der väterlichen Werkstatt zu den akademischen Titeln. Ihre Tätigkeit zwischen Wien, Paris, Prag, Athen und der deutschen Stadt Kaiserslautern war bald durch den frühen Tod der beiden unterbrochen. Ihr um wenige Jahre jüngerer Landsmann aus Poljanska dolina bei Bischofslack (Škofja Loka), Anton Ažbe (1862-1905), malte nach seinem Studium in Wien relativ wenig, dafür gründete er aber in München seine berühmte Privatschule, die auch einige spätere führende europäische Maler besuchten, wie beispielsweise V. Kandinsky, A. Javlensky, L. Kuba und einige andere. Damit schrieb er sich für alle Zeiten in die Geschichte der mitteleuropäischen Malerei ein. 
Einen neuen Aufschwung in Richtung der internationalen Horizonte erlebte die slowenische Kunst erst während der Sezessionszeit. Die Architekten Maks Fabiani (1865-1962), in dessen Adern viel slowenisches Blut kreiste, und Jože Plečnik (18721957) beteiligten sich nach ihren Ausbildungsjahren in der Hauptstadt ÖsterreichUngarns aktiv an der Erneuerung der Kunst. Beide wirkten gerade in der Schlüsselphase der Wiener Moderne mit, als ein Kreis der Architekten unter der Führung von Otto Wagner mit dem Historismus brach und den Weg für den späteren Stil des Funktionalismus bereitete. Das Wiener Gebäude Portois \& Fix von Fabiani und das Zacherl-Haus von Plečnik, in der Nähe des Stephansdoms, stellen zwei extreme Entwicklungsmöglichkeiten der Sezessionsarchitektur dar. Am Stadtrand von Wien ging Plečnik noch einen Schritt weiter mit der Heiliggeistkirche, einem der ersten sakralen Eisenbetonbauten in der Welt. Nach dem Ersten Weltkrieg erreichte er den Gipfel seines Schaffens in Böhmen mit dem Umbau der Prager Burg für den ersten tschechoslowakischen Präsidenten Tomaš Garrigue Masaryk. Im Vergleich zu seinen Erfolgen scheinen relativ bescheiden die allerdings unumstrittenen Erfolge der Gruppe der Impressionisten, in der neben ihrem Leiter Rihard Jakopič (1869-1943) noch Ivan Grohar (1867-1910), Matija Jama (1872-1947) und Matej Sternen (1870-1949) waren, obwohl gerade diese Maler später die slowenische Kunst zwischen den beiden Kriegen bestimmten. Plečniks Arbeit erreichte Weltgeltung besonders nach der großen Pariser Ausstellung im Jahre 1986.

Während der Zeit zwischen den beiden Weltkriegen verlor Wien seinen ehemaligen Charme für die slowenischen Künstler und sie bevorzugten es, an der Prager oder Agramer (Zagreber) Akademie der bildenden Künste zu studieren. Nur der Triester Avgust Černigoj (1898-1985), der stärker mit der italienischen Avantgarde verbunden war, machte seine Ausbildung in München und am Weimarer Bauhaus. Der Begriff Mitteleuropa verlor nach dem Zweiten Weltkrieg durch die Teilung des Kontinents auf zwei Blöcke seine Bedeutung und wurde für die politischen Machthaber sogar politisch verdächtig. Allerdings blieb Jugoslawien formell außerhalb der genannten Teilung, aber es öffnete sich lange nicht gegenüber dem Westen. Das Geschehen hinter dem Eisernen Vorhang war uninteressant für die slowenischen Künstler; sie versuchten eher, sich an Paris und andere Metropolen der Weltkunst anzunähern. Einer der wenigen, die international berühmt wurden, ist unser in Wien lebender Landsmann, der Architekt Boris Podrecca (geb. 1940).

Nach dem Fall der Berliner Mauer lebte auch das ehemalige Mitteleuropa auf, aber die moderne slowenische bildende Kunst orientierte sich noch lange nach den Vereinigten Staaten Amerikas. Erst in der neueren Zeit kommt es erneut zur Annäherung an die Länder des deutschen Sprachraumes. Das slowenische Sich-Öffnen verläuft aber hauptsächlich auf der Ebene der alternativen Kunst, deren Ausdrucksmittel vorwiegend nicht zur bildenden Kunst gehören. Deswegen ist es sehr schwer zu sagen, wie fest wir Slowenen in der Tat heute noch in der mitteleuropäischen Kultur verankert sind, auf deren Tradition alle unsere größten Errungenschaften gründen. 
MUZIKOLOŠKI ZBORNIK - MUSICOLOGICAL ANNUAL XL

\section{Hinweis zur Literaturauswahl}

Der erste, der den Anteil der heimischen Kreativität und die Verbundenheit der letzteren mit Mitteleuropa und Italien zeigte, war France Stele in seinem Überblick uber die slowenische Kunst (Oris zgodovine umetnosti pri Slovencih [Umriß der Kunstgeschichte der Slowenen], Ljubljana 1924). Obwohl er später seine Erkenntnisse in vieler Hinsicht ergänzte, stellt das erwähnte Buch hinsichtlich der Frage unserer kulturellen Verankerung im mitteleuropäischen Raum noch immer einen nützlichen Führer dar. Besonders viel Beachtung fand Steles These von den regionalen Konstanten der slowenischen Kunst, die die Gesetzmäßigkeiten des Ausdruckes in der bildenden Kunst einzelner slowenischer Regionen behandelt. Am konsequentesten berücksichtigt wurde diese These von Nace Šumi bei seiner Erforschung der Barockarchitektur (Ljubljanska baročna arhitektura [Laibacher Barockarchitektur], Ljubljana 1961; Baročna arhitektura [Barockarchitektur], Ljubljana 1969). Detailliert befaßte sich mit den Fragen der Konstanten und der mitteleuropäischen Einflüsse auf die slowenische bildende Kunst Emilijan Cevc in seinem Übersichtsbuch Slovenska umetnost [Slowenische Kunst] (Ljubljana 1966) und bei seinen Erforschungen der mittelalterlichen und frühbarocken Plastik (Srednjeveška plastika na Slovenskem [Mittelalterliche Plastik in Slowenien], Ljubljana 1963; Poznogotska plastika na Slovenskem [Spätgotische Plastik in Slowenien], Ljubljana 1970; Kiparstvo na Slovenskem med gotiko in barokom [Bildhauerkunst in Slowenien zwischen Gotik und dem Barock], Ljubljana 1981). Ein neuer Versuch des ganzheitlichen Überblicks der slowenischen Kunst wurde von Nataša Golob herausgegeben, unter Mitwirkung von mehreren anderen Autoren (Umetnost na Slovenskem [Kunst in Slowenien], Ljubljana 1998). Mit der Bildhauerkunst der slowenischen Steiermark und deren Verbundenheit mit dem österreichischen Teil des genannten Landes befaßte sich Sergej Vrišer (Baročno kiparstvo na slovenskem Štajerskem [Barocke Bildhauerkunst in der slowenischen Steiermark], Maribor 1963). Anica Cevc war die erste, die auf die Verbindungen mit der österreichischen Steiermark im Rahmen der Barockmalerei aufmerksam machte (insbesondere in: Anton Lerchinger, Zbornik za umetnostno zgodovino, Neue Reihe V-VI, Ljubljana 1959). Im Jahre 1992 organisierten "Zveza zgodovinskih društev Slovenije" und das österreichische "Institut für Ost- und Südosteuropaforschung" ein Symposium zum Thema "Wien und die Slowenen" (Dunaj in Slovenci"), worüber der gleichnamige Symposiumsbericht veröffentlicht wurde (Ljubljana 1994). Janez Höfler studierte die Kärntner Wandmalerei und ihre Verbundenheit mit dem Land Krain (Die gotische Malerei Villachs: Villacher Maler und Malerwerkstätten des 15. Jahrhunderts, Villach 1981 und 1982). Er war auch einer der Initiatoren der Ausstellung der gotischen Kunst in Slowenien und des darauffolgenden internationalen Symposiums (Gotika v Sloveniji - Gotik in Slowenien - Il gotico in Slovenia, Ljubljana 1994). Außerdem veröffentlichte Höfler unlängst eine Anthologie mit Beiträgen von ausländischen und inländischen Forschern über die Verbindungen zwischen der slowenischen und der bayerischen Kunst im Mittelalter (Bayern und Slowenien in der Früh- und Spätgotik, Regensburg 2003). Unter den jüngeren Forschern, die sich den mitteleuropäischen Problemen der slowenischen Kunst widmen, muß man Barbara Murovec erwäh- 
MUZIKOLOŠKI ZBORNIK - MUSICOLOGICAL ANNUAL XL

nen (Likovni viri za baročno stropno slikarstvo v Sloveniji [Kunstquellen für die Deckenmalerei des Barocks in Slowenien], Zbornik za umetnostno zgodovino, Neue Reihe XXXIX, Ljubljana 2003). 